\title{
An Assessment of Survival Rates and Within-patient Clustering of Failures for Endosseous Oral Implants
}

\author{
R.J. WEYANT and B.A. BURT ${ }^{1}$
}

Department of Public Health and Community Dentistry, School of Dental Medicine, University of Pittsburgh, Pittsburgh, Pennsylvania 15261 and VA Medical Center, Highland Drive, Pittsburgh, Pennsylvania; and ${ }^{1}$ School of Public Health, The University of Michigan, Ann Arbor, Michigan 48109-2029

This study examined endosseous cylinder implant survival, defined as the unqualified presence of the implant in the mouth at the end of the observation period, in 598 consecutive VA patients, with a total of 2098 implants. Data were taken from the Department of Veterans Affairs (VA) Dental Implant Registry, which has maintained longitudinal data on the survival of individual dental implants in VA patients since 1987. The maximum time of observation in any one patient was 2040 days ( $5.6 \mathrm{yr}$ ). Survival analysis by use of life-table methods was carried out on both an implant- and a patient-specific basis. Implant cases were accrued randomly, and therefore a random censoring model was used. A correlated binomial model was used for assessment of the degree of withinpatient clustering of implant removals. Results showed that the implant-specific survival rate during the longest time interval $(5.6 \mathrm{yr})$ was $89.9 \%$; the patient-specific implant survival rate during the same time was $78.2 \%$. Among implants which were removed, the mean time to removal was 292 days. The hazard function, which describes the probability of implant loss as a function of time, decreased steadily throughout the observation period. The correlated binomial model suggested a clustering of removals within patients with multiple implants $(\rho=0.11, p=0.0001)$. The odds of having a second implant removed were 1.3 times greater if the patient had already had one implant removed.

This study suggests that when implants fail, they do so soon after placement, and the likelihood of failure decreases steadily from implantation through the first five years post-surgery. These findings also suggest the existence of systemic factors which affect the survival of all implants within a given patient and may lead to multiple implant failures. Identification of these systemic factors may lead to improvements in patient selection and increases in the success rates for oral implants. Finally, results show that pure titanium implants had better short-term survival rates, but worse long-term survival rates, when compared with implants that had some other form of surface coating.

\section{J Dent Res 72(1):2-8, January, 1993}

\section{Introduction.}

Recent trends indicate that clinicians and patients alike are no longer thinking of implants as experimental or as

Received for publication March 3, 1992

Accepted for publication July 31, 1992

Based on a dissertation submitted to the graduate faculty, University of Michigan School of Public Health, in partial fulfillment of the requirements for the $\mathrm{DrPH}$ degree methods of last resort, but rather as a first-choice treatment option (McKinney et al., 1988; Worthington, 1988). Schnitman and Shulman (1979) summarized the current status of implants when they stated, "Aggressive marketing of new materials and techniques has resulted in significant public demand, driving these new technologies into uncontrolled widespread clinical use." The recency of this trend is reflected in the lack of relevant literature reporting on outcomes. In addition, demographic changes, changing expectations for oral health, and the fact that many millions of people still have incomplete dentitions probably mean that the demand for dental implants will increase (Meskin and Brown, 1988).

In spite of a dearth of research to support efficacy, many oral implant systems are approaching a presumptive level of acceptable effectiveness in the eyes of many clinicians, even when efficacy claims remain unsupported by objective research. When they do exist, efficacy studies of some implant systems report equivocal results. A general lack of standardization and poor study design permeate most reports, with some authors concluding that the majority of studies fall far short of meeting acceptable standards for a clinical trial (Kapur and Garrett, 1988). Kapur and Garrett (1988) correctly attributed the confusing literature to a lack of well-designed, independent studies that objectively examine implant performance and concluded, "A reader cannot help thinking that many of these reports are efforts to promote a given system, rather than to present reliable scientific information based upon sound study design."

In practice, many clinicians, guided primarily by clinical judgment, select a variety of implant types to address the various clinical problems found in their patients. For this reason, it is important to characterize what overall outcomes can be expected from the routine use of implants in a variety of clinical settings. There are currently no studies that report on the nature and frequency of implant outcomes experienced in routine practice settings. Even when they attempt to use representative populations, implant studies often exclude certain patients and providers in a way that might tend to favor implant success, possibly inflating success rates when compared with implants placed under less restrictive conditions (Kapur, 1987; van Steenberghe et al., 1990). It is possible and perhaps even likely that the reported survival rates from many current studies are quite different from what might be expected from implants placed in more routine clinical settings. Similarly, variation in definitions of success can result in significant variations in reported outcome rates. Malmqvist and Sennerby (1990), for example, arrived at the different success rates of $37.2 \%$ and $9.3 \%$ when they applied two commonly accepted implant success criteria to the same patient population. One way to reduce 
implant survival were examined between pure titanium implants and other types of implants and as a function of the time of implant placement.

\section{Materials and methods.}

The data source for this study was the Department of Veterans Affairs (VA) Dental Implant Registry. The VA began offering dental implants to eligible patients on a routine basis in 1987. The Dental Implant Program within VA authorizes VAMC (VA Medical Center) dental services to place dental implants in veterans, and requires the submission of reports on all implant cases to VA's Dental Implant Registry. The implant registry provides for the collection of data on all aspects of the construction of an implant-borne prosthesis. To accomplish the data collection, dentists at authorized VAMC implant sites complete and submit data collection forms for each implant case that summarize details and complications of implant surgery, prosthodontic restoration, and recall follow-up of the patient. When necessary, the date of implant removal is also recorded in the registry. At the time of the study, there were 61 VAMC's $(35 \%)$ with approved implant programs, with 49 facilities reporting at least one implant case to the registry. This study included all endosseous cylindrical (root-form) implants contained in VA's Dental Implant Registry which had at least one post-surgical follow-up examination as of 31 December 1990.

Registry data were maintained on microcomputers with dBase III Plus software. All data, prior to computer entry, were checked for accuracy by an independent dentist associated with the registry. All data analysis was done by use of SAS-PC software.

Study variables.-Implant failure, defined here as the unqualified removal of the implant from the dental arch, was the major endpoint considered in this study. This study did not consider implants that were "submerged", or buried within the patient's bone, and were allowed to remain in situ. The length of survival was measured in days from initial implantation to removal. All root-form implants used in this study required a two-stage surgical implantation procedure.

In addition to an overall survival rate, several stratifying variables were included to determine whether they contributed to implant survival. The date of the implant surgery was used to assess whether implants that had been placed earlier in the Program had survival rates different from those of implants which had been placed later. Information on each implant's surface material was used to determine whether survival of pure titanium implants differed from that of implants with other surface-coating materials. The construction of these variables is described below.

Time of implant placement [EARLY].-A dichotomous variable (EARLY) was created that divided implants into the categories of those implants placed before January 1 , 1988 (EARLY = 1), and those placed on or after January 1,1988 (EARLY $=0$ ). This date was selected since it represented the approximate midpoint of the data in the registry.

Implant surface coating [BOND].-Implant surface coatings provide the physical-chemical interface with bone that facilitates osseointegration. Based on surface- covering material, a dichotomous variable (BOND) was created which classified implants into two groups: implants with a pure titanium surface $(B O N D=0)$ and all other implants $(B O N D=1)$. Pure titanium has low antigenicity and yet provides a biologically suitable site for bone adaptation. Various other materials have been used on implants as a way of increasing the surface area or of improving the bioactivity of the implant surface in an attempt to provide a greater degree of bone adaptation. The other coatings, however, may have a tendency to delaminate or to trap oral debris and bacteria at a higher rate than titanium implants. A variety of coating materials has been used in implant manufacturing. Those in the registry included ceramic, sapphire crystal, and hydroxylapatite (HA) coatings. Over $90 \%$ of the "coated" implants were HA-coated.

Incidence of implant failure.-The registry consists of longitudinal data; patient data are entered periodically as treatment progresses. Patients can stop contributing data to the registry at various times due to loss from follow-up or implant removal. Incidence density (ID) is an appropriate measure for estimation of the rate of occurrence of various outcomes in a dynamic population (Kleinbaum et al., 1982). Incidence densities were obtained by dividing the number of implant losses by the total population-time of follow-up. Here, individual implants were considered as the unit of analysis, so that an ID assessment for each implant category would provide an overall occurrence rate for implant losses per unit of implant-time (i.e., implant-day).

Stratification of ID calculations over selected time intervals allowed an assessment of the time-dependency of implant losses to be made. The ID calculations were stratified into the time intervals of 0-120 days, 121-360 days, and greater than 360 days - categories which roughly approximated the submission patterns of forms to the registry. Stratum one (0-120 d) represents the average time from implant surgery to prosthesis placement, the second stratum (121-360 d) corresponds approximately to the early post-prosthesis phase, and the final stratum (> $360 \mathrm{~d})$ represents the long-term healing period and frequently corresponds to the placement of the patient into a routine (semi-annual) recall program. The ID was calculated within each of the three time intervals by the following formula:

$$
\mathrm{ID}=\mathrm{X} / \sum \Delta \mathrm{T}_{\mathrm{i}}
$$

where $\mathbf{x}$ is the number of implant losses during the specified interval, and $\Delta T_{i}$ is the duration of the observed followup for the ith implant from entry into the study until the implant is either removed or censored. Censoring can occur when either the patient is lost to follow-up or the end of the study period occurs and the implant has not failed.

Incidence density ratios (IDR) were used to compare each of the various groupings of implants by use of a large sample $\mathrm{z}$-test to determine p-values (Kleinbaum et al., 1982). The IDR tests the hypotheses H0: IDR = 1 vs. HA: IDR $\neq 1$ for two groups of implants ( 1 and 2), as expressed in the following formula:

$$
\mathrm{IDR}=\mathrm{ID}_{1} / \mathrm{ID}_{2}
$$

The large sample z-test used for computation of an 
TABLE 1

DEMOGRAPHIC CHARACTERISTICS OF STUDY POPULATION

Mean age

$58.7 \mathrm{yr}$

Age range

19-77 yr

Percent distribution

by age strata

Under $40 \mathrm{yr}$

$40-49$ yr

10.5

$50-59$ yr

$60-69 \mathrm{yr}$

$70 \mathrm{yr}$ or older

Percent male

Percent female

Percent distribution

by ethnic identification*

Black

Caucasian

Hispanic

Other

0.4

\footnotetext{
${ }^{*}$ Category names are as they appeared on data collection forms.
}

approximate p-value was calculated with the following formula (Kleinbaum et al., 1982):

$$
z=a-(m)(p) / \sqrt{ }(m)(p)(1-p)
$$

where:

$\mathrm{a}=$ the number of implant losses in group 1 ,

$\mathrm{m}=$ the total number of implant losses in groups 1 and

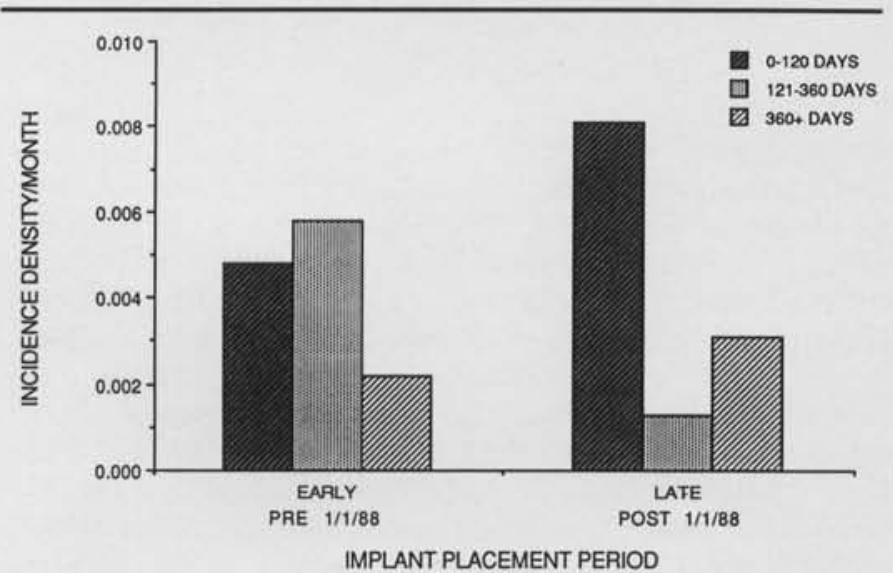

Fig. 1-Incidence density of implant losses stratified by time period of implant surgery (EARLY) for three post-surgical time intervals $($ Early $=$ implants placed before $1 / 1 / 88$; Late $=$ implants placed on or after $1 / 1 / 88$ ).
TABLE 2

RESULTS OF CORRELATED BINOMIAL MODEL FOR IMPLANT FAILURE

\begin{tabular}{lcc}
\hline \hline & \multicolumn{2}{c}{ Class Variable } \\
\cline { 2 - 3 } & Patient & Provider \\
\hline Binomial proportion & 0.062 & 0.062 \\
Correlated binomial & & \\
proportion & 0.061 & 0.061 \\
Rho-coefficient & 0.114 & 0.01 \\
Significance & 0.001 & 0.001 \\
\hline
\end{tabular}

$\mathrm{p}=$ the proportion of total implant-time contributed by implant group 1.

Survival probabilities.-Life-table methods were used for estimation of implant survival probabilities while censored observations were controlled for. Implants that had not been removed from the mouth were considered as randomly censored by the end of the data collection phase. Overall implant-specific and patient-specific survival estimates were made with 120-day intervals used up to 1040 days $(5.6 \mathrm{yr})$, the maximum reported time to follow-up for any implant. Additional stratified analyses were carried out with surface coating (BOND) and time of implant placement (EARLY) used as the stratifying variables. The logrank and Wilcoxon tests were used for assessment of significant differences among the strata (Lawless, 1982).

Both survival times and a hazard function were calculated. The hazard function provides an estimate of the instantaneous likelihood of loss of the implant during any interval.

Correlated binomialmodeling.-With implant survival as the event of interest, the implant-specific data were analyzed for evidence of within-patient and within-provider correlations by means of a correlated binomial model. The rho statistic ( $\rho$ ) generated from this model ranges between -1 and 1 . A value near zero is evidence of no within-patient correlation, with evidence for correlation increasing as the rho statistic moves toward 1 (Kupper and Haseman, 1978).

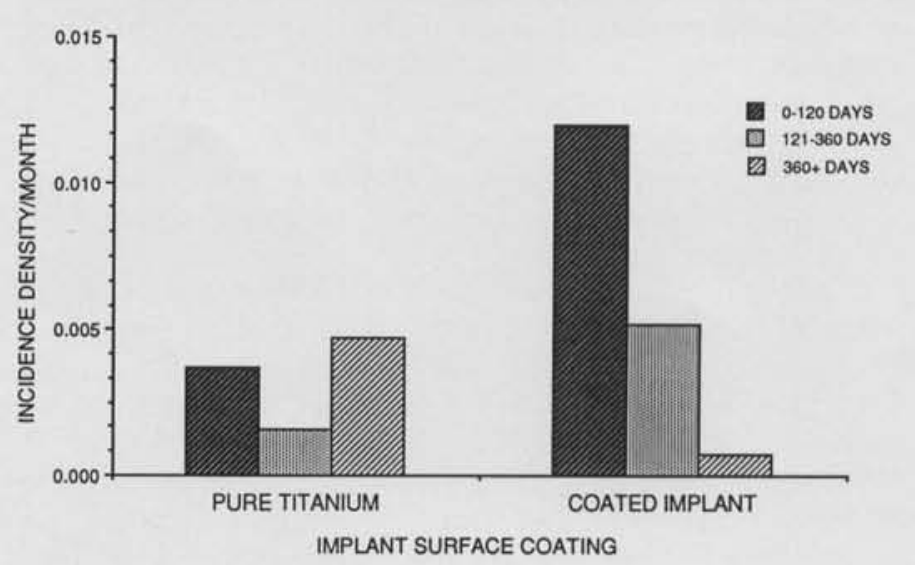

Fig. 2-Incidence density of implant losses stratified by implant coating (BOND) for three post-surgical time intervals. 


\section{Results.}

The selection criteria (root-form implants, at least one post-surgical follow-up appointment) were met by 598 patients in whom 2098 implants had been placed. Demographic information for the study population appears in Table 1. The study population consisted predominantly of white males, with the 60-69-year-old age strata constituting the largest single group receiving implants (46.5\%), reflective of veteran cohorts which commonly seek other medical services (Department of Veterans Affairs, 1989).

Implant removals were rare, with 81 implants in 45 subjects removed, resulting in an average of $1.8 \mathrm{implant}$ removals per "failed" case. This resulted in a crude rate for implant survival of $96.1 \%$ for the duration of the study, with $92.4 \%$ of subjects having no implants removed. There were 61 cases where the subject received only one implant, with three of these implants failing (4.9\%). No multiple-implant case had more than four implant removals, but $57 \%$ of all multiple-implant cases with at least one removal had two or more removals, resulting in odds of 1.3 for having a second implant removal, given that one implant had already been removed.

Correlation of implant failures.-The results of the correlated binomial model analyses are given in Table 2, which provides estimates of $\rho$ and an adjusted binomial proportion for implant loss on an implant- and providerspecific basis. In both cases, rho showed a high degree of statistical significance, based on likelihood ratio tests.

Incidence densities for three post-surgical periods.Figs. 1 and 2 provide the results of the stratified ID calculations for implants during the three post-surgical time periods. For BOND, significant differences $(p<0.01)$ were found in the pre-prosthetic period (0-120 d) and in the long-term period ( $>360 \mathrm{~d}$ ), but not in the intermediate period. Implants placed prior to 1988 (EARLY) showed significantly worse survival rates during the intermediate period (121-360 d) than did implants placed after $1 / 1 /$ 88 , but no significant differences were found during the other time intervals.

A stratified analysis was carried out to examine for the possibility of confounding among the EARLY time period, implant coating, and implant brand. If one type of implant was used preferentially in the pre- 1/1/88 period,



Fig. 3-Implant- and patient-specific survivor functions. then these implants would have been at risk for a longer time, thereby increasing the probability of loss. A chisquare analysis provided no evidence of confounding for pure-titanium vs. "coated" implants $(p=0.42$ ) or for differences in implant brand ( $p=0.63$ ) in either time period.

Implant survival probabilities.-Time to implant removal (failure) was available for all implants, with the mean time to removal for lost implants being 292 days. The overall implant-specific and patient-specific survival probabilities for the complete study population are provided in Fig. 3. The implant-specific survival probabilities during the last 120-day interval (1920-2040 days) was $89.9 \%$. This survival rate was first reached at 1080 days $(2.9 \mathrm{yr})$. The patient-specific analysis represents the probability of at least one implant removal within a patient. The overall survival probability for patientspecific implant removals over the same time interval was $78.2 \%$.

The overall hazard function for all implants placed in the study population is provided in Fig. 4. In addition to the resultant hazard function calculated at each interval, a second-order polynomial curve was fit to the data by a least-squares procedure. The resultant curve suggests that the probability of implant loss decreased steadily from the time of implantation through the duration of the observed data (2040 d).

Additionally, the data were explored for significant differences in survival based on differences in surface coating (BOND) and time period of placement (EARLY). No difference was detected between the time period of implant placement and survival. The stratified analysis for implant coating (BOND) is plotted in Fig. 5. Both the logrank $(p=0.02)$ and the Wilcoxon $(p=0.003)$ tests indicated that significant differences in survivor functions existed between pure titanium and all other implants. The mean time to removal reported for "coated" implants was 149 days, while for pure-titanium implants which were removed, the mean time until removal was 452 days.

\section{Discussion.}

This longitudinal study of dental implants was made possible by the 1987 creation of VA's dental implant

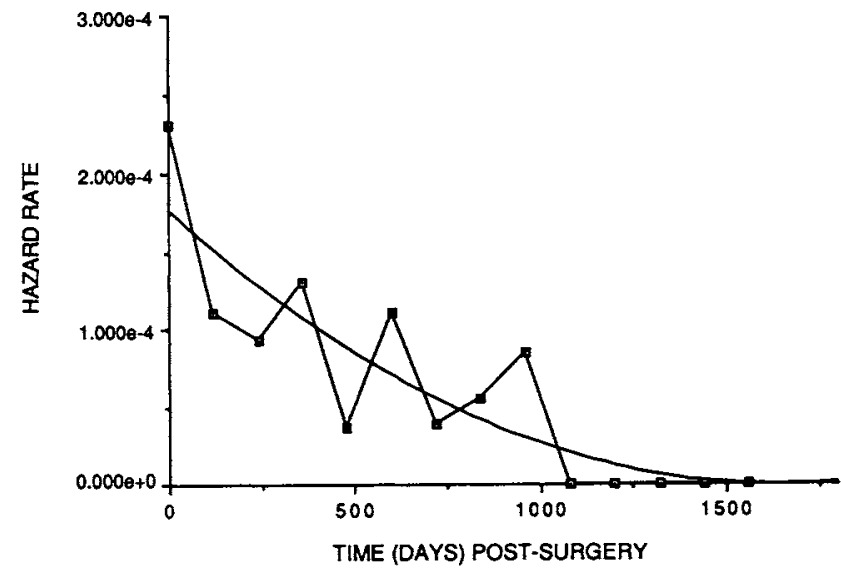

Fig. 4-Overall hazard rate for implant survival. 


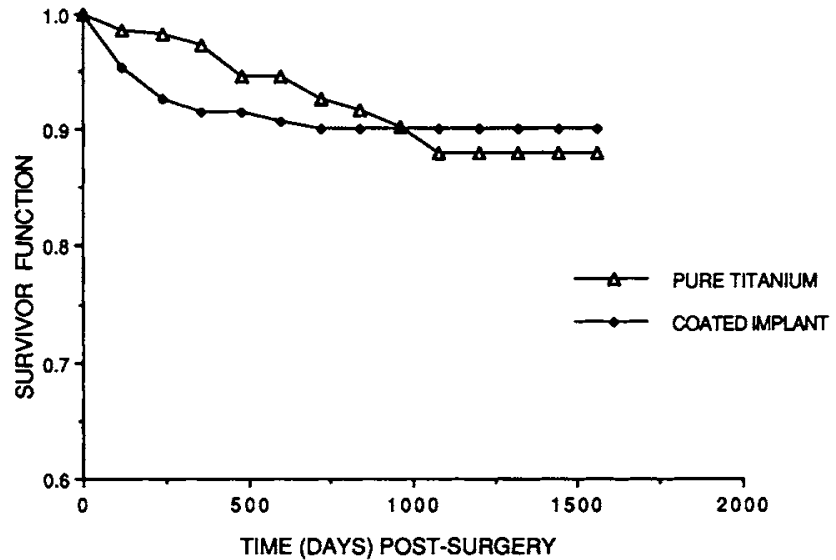

Fig. 5-Survivor function for implants stratified by surface coating (BOND).

registry. Although not set up for experimental studies, the registry allows for the creation and efficient analysis of a large number of variables. There are inherent and well-recognized limitations to panel studies that derive from the nature of their design. The major limitations are the lack of experimental design and potential for information bias (Cook and Campbell, 1979). Nonetheless, the value of appropriate analysis of the registry far outweighs these limitations as long as they are recognized.

The absence of subject and implant randomization and control groups limits the testing of hypotheses about implant survival. A clinical trial, however, evaluating a large number of implant systems and many cofactors, would be preclusively expensive without some way of guiding hypothesis generation. This study should be viewed as a necessary first step to the creation of hypotheses that can lead to more efficient clinical trials.

The extent of selective reporting and misclassification of cases has not been assessed for the registry data. Several features of the registry, however, work to minimize selective reporting. These features include mandatory reporting requirements and review of reported cases by supervisory personnel. Misclassification error is a problem in studies where data are collected from many examiners, especially when detailed assessments are made by noncalibrated examiners. To minimize misclassification, we used the easily applied criteria of implant type and implant removal as our primary variables of interest. Both of these variables require little subjective evaluation and are probably accurately and consistently reported in the majority of cases. The scope of the registry precludes examiner calibration to the level necessary for reliable application of some of the commonly reported measures of implant health, other than implant removal, and analysis of registry data by more detailed criteria would probably result in a study with much lower validity.

Studies comparing implant performance across brands represent one of the most compelling areas for further implant research. Comparative studies of cylindrical implant performance as a function of brand, however, are absent from the literature, and variations in study design make comparisons among studies difficult. Implant performance, as it relates to subsequent prosthesis design, and implant placement in the arch are also areas in need of more detailed analysis. This study did not focus on brand, prosthesis design, or implant placement, since adequate characterization of the effects of these factors on implant removal rates, even in a study of as many cases as this one, would have resulted in over-stratification of the data. For that reason, we resisted the urge to run analyses on every conceivable subgroup. As the registry accrues more cases, however, it will be able to support detailed investigations of the roles of prosthesis and implant design. Since factors such as implant design and placement in the arch can clearly act as confounders, however, we explored the effects of implant design, patient age, and arch placement and found no evidence that these factors were acting as significant modifiers of the overall rate of implant loss.

This study probably represents well the VA patient population heretofore receiving root-form dental implants. Although VA patients are not representative of the general US population, this group of veterans, characterized by multiple medical problems and high rates of alcohol and tobacco use, may well represent one of the most challenging groups in terms of implant survival. Findings in this patient group may well depict conservative estimates of implant performance if, as suggested, systemic medical factors play a significant role in implant health.

As reported above, a highly significant within-patient correlation for implant loss $(\rho=0.11, p=0.0001)$ was found. The interpretation of this finding is not straightforward. Theoretically, $\rho$ can range from -1 to 1 , with values near zero indicating little correlation or clustering of outcome within levels of the class variable. High positive values of $\rho$ suggest that some type of clustering is occurring. In this case, high $\rho$ values would mean that a subject who has experienced one implant removal is more likely to experience a second. As an aid in interpretion of the magnitude of these findings, it should be noted that rho's permissible values are mathematically constrained by the maximum number of implants placed in any subject (Kupper and Haseman, 1978). This study had a maximum of 14 implants, and results in a maximum rho of approximately 0.13 . The finding of this study $(\rho=0.11)$, therefore, is approaching the mathematical maximum for these data.

This large rho statistic-along with the finding that a patient is 1.3 times more likely to have a second implant removed, given that he has had one removed-is evidence that within-patient or systemic factors are important in characterization of implant losses. Systemic factors are factors which affect all of the implants within an individual, such as medical, implant, provider, or treatment facility factors. This study adds to the evidence from other studies (Adell et al., 1981; Babbush et al., 1986; Cox and Zarb, 1987; Ahlqvist et al., 1990) that certain patients are at comparatively higher risk for loss of implants than others, suggesting that future research should be directed at identifying high-risk patients.

The within-provider assessment showed little correlation of implant removals attributable to providers. A $\rho$ of 0.01 indicates that very little of the variation in implant outcomes was attributable to provider differences, and would be consistent with a conclusion that there is little evidence in this study of clustering of implant removals among individual dentists. It has been argued that the success of oral implants is dependent on operator skill 
(Worthington et al., 1987). This study does not refute that contention, but rather has found that within VA there is not one dentist or group of dentists who are responsible for a disproportionate number of lost implants among their cases.

In addition to the clustering of implant losses within patients, these results suggest that implant removal rates are highest immediately following surgery and then decrease for the next five years. In the stratified analysis, the early post-implant period (0-120 d) had the highest incidence density rate, a finding which is reflected in the decreasing hazard function (Fig. 4). High implant loss rates early in the post-surgical period have been reported by others (Adell et al., 1981; Babbush et al., 1986; Cox and Zarb, 1987; van Steenberghe et al., 1990). None of these studies, however, accounts for implant censoring in their survival estimates, and therefore no reliable estimate of the hazard rate can be made. It is quite possible that the hazard rate will begin to increase as longer observation times occur, since a decreasing then increasing hazard function is a common "failure" pattern in biological and mechanical systems (Lawless, 1982). The declining hazard rate through five years has implications for those designing future clinical trials. Due to the relatively high rate of implant loss in the early post-surgical period, it may be possible to sort through the effects on survival of certain variables with relatively short follow-up studies. In fact, these results support a five-year survival rate as a reasonable duration in which to estimate implant performance. However, it is always dangerous to extrapolate beyond the range of the data; consequently, additional monitoring of the Registry for changes in the hazard rate are indicated.

There was no evidence that implants placed earlier in the Implant Program were at higher risk for removal than more recently placed implants when comparisons were made during similar post-surgical time intervals. Implant coating (BOND), however, did seem to alter implant survival rates. Coated implants performed significantly worse only in the early period (0-120 d). There was some (non-significant) evidence that coated implants performed less well in the intermediate period (121-360 d), and then significant evidence of a reversal in trends over the long term ( $>360 \mathrm{~d}$ ). Since this study was exploratory, finer distinctions between types of implant coatings were not made. The majority of coated implants were HA-coated (> $90 \%$ ), however, suggesting that future studies of the effects of HA coatings may be indicated.

The use of survival analysis techniques provided several benefits over other methods by: (1) taking full advantage of the information on implants which had not yet failed, (2) providing a dynamic model of the events which can lead to possible prognostic determinations of implant survival times, and (3) correctly adjusting survival probabilities within each interval to account for lost and censored data. The life-table analysis demonstrates how the censoring of observations can change survival probability estimates ( $96.0 \%$ vs. $89.9 \%$ for implant-specific survival). Even more dramatic was the difference found in the patient-specific implant survival rates $(92.4 \%$ vs. $78.2 \%$ ). Failure to account for censored data results in the over-estimation of survival probabilities, an apparent problem in several recent studies (Cox and Zarb, 1987; Albrektsson et al., 1988; Patrick et al., 1989).
Although clinicians and patients might be re-assured from the finding that only about $10 \%$ of all implants were removed, the patient-specific removal rate of $78 \%$ means that nearly one-quarter of all patients experienced at least one implant loss. The clinical significance of this depends on the severity of the sequellae associated with implant removal, but high implant-specific success rates may be misleading to patients when they evaluate the probability of experiencing implant-associated morbidity.

Given that the patient population in this study was likely to have more medical complications and to be older than many groups receiving implants in the US, this study would suggest that an overall implant survival rate in excess of $90 \%$ for five years could be expected in many patient populations. The clustering of implants, however, would indicate that certain systemic factors play an important role in determining whether a particular patient will be able to tolerate implants. Future research should be directed at uncovering these systemic factors, thereby improving patient selection and subsequent implant success rates. In addition, longer time periods of observation are indicated to determine whether the declining implant loss rate reverses after five years. Finally, the role of implant coatings as they relate to survival should be explored in a clinical trial.

\section{Acknowledgments.}

The authors extend their gratitude to the Department of Veterans Affairs' Office of Dentistry and all VA Medical Center personnel involved in the Implant Program for their support of this study. We also thank Dr. Craig Misch for his helpful comments during the writing of this paper.

\section{REFERENCES}

Adell R, Lekholm U, Rockler B, Brånemark P-I (1981). A 15-year study of osseointegrated implants in the treatment of the edentulous jaw. Int J Oral Surg 10:387-416.

Ahlqvist J, Borg K, Gunne J, Nilson H, Olsson M, Astrand P (1990). Osseointegrated implants in edentulous jaws: a 2-year longitudinal study. Int $J$ Oral Maxillofac Implants 5:155-163.

Albrektsson T, Dahl E, Enbom L, Engevall S, Engquist B, Eriksson AR, et al. (1988). Osseointegrated oral implants: a Swedish multicenter study of 8139 consecutively inserted Nobelpharma implants. $J$ Periodontol 59:287-296.

Babbush CA, Kent JN, Misiek DJ (1986). Titanium plasma-sprayed (TPS) screw implants for the reconstruction of the edentulous mandible. J Oral Maxillofac Surg 44:274-282.

Cook TD, Campbell DT (1979). Quasi-experimentation design and analysis issues for field settings. Chicago (IL): Rand-McNally.

Cox JF, Zarb GA (1987). The longitudinal clinical efficacy of osseointegrated dental implants: a 3-year report. Int $J$ Oral Maxillofac Implants 2:91-99.

Department of Veterans Affairs (1989). 1987 survey of veterans, DM\&S M-70-89-1. Washington (DC): US Bureau of the Census.

Engquist B, Bergendal T, Kallus T, Linden U (1988). A retrospective multicenter evaluation of osseointegrated implants supporting overdentures. Int J Oral Maxillofac Implants 3:129-134.

Jemt T, Lekholm U, Adell R (1989). Osseointegrated implants in the treatment of partially edentulous patients: a preliminary study on 876 consecutively placed fixtures. Int $J$ Oral Maxillofac Implants 4:211-217.

Kapur KK (1987). Veterans Administration cooperative dental 
implant study - comparisons between fixed partial dentures supported by blade-vent implants and removable partial dentures. Part I: methodology and comparisons between treatment groups at baseline. J Prosthet Dent 58:499-511.

Kapur KK, Garrett NR (1988). Requirements for clinical trials. $J$ Dent Educ 52 (Spec Iss):760-764.

Kleinbaum DG, Kupper LL, Morgenstern H (1982). Epidemiological research: principles and quantitative methods. New York: Van Nostrand Reinhold.

Kupper LL, Haseman KJ (1978). The use of a correlated binomial model for the analysis of certain toxicological experiments. Biometrics 34:69-77.

Lawless JF (1982). Statistical models and methods for lifetime data. New York: John Wiley and Sons.

Malmqvist JP, Sennerby L (1990). Clinical report on the success of 47 consecutively placed Core-Vent implants followed from 3 months to 4 years. Int $J$ Oral Maxillofac Implants 5:53-60.

McKinney RV, Steflik DE, Koth DL, Singh BB (1988). The scientific basis of dental implant therapy. J Dent Educ 52:696-705.

Meskin LH, Brown JB (1988). Prevalence and patterns of tooth loss in US employed adult and senior populations. $J$ Dent Educ
52:686-691.

Naert I, DeClerq M, Theuniers G, Schepers E (1988). Overdentures supported by osseointegrated fixtures for edentulous mandible: a 2.5 year report. Int J Oral Maxillofac Implants 3:191-196.

Patrick D, Zosky J, Lubar R, Buchs A (1989). The longitudinal clinical efficacy of Core-Vent dental implants: a five year report. J Oral Implantol 15:95-103.

Schnitman PA, Shulman LB (1979). Recommendations of the consensus conference on dental implants. J Am Dent Assoc 98:373-377.

van Steenberghe D, Lekholm U, Bolender C, Folmer T, Henry P, Herrmann I, et al. (1990). The applicability of osseointegrated oral implants in the rehabilitation of partial edentulism: a prospective multicenter study on 558 fixtures. Int $J$ Oral Maxillofac Implants 5:272-280.

Worthington P (1988). Current implant usage. J Dent Educ 52 (Spec Iss):692-695.

Worthington P, Bolender CL, Taylor TD (1987). The Swedish system of osseointegrated implants: Problems and complications encountered during a 4-year trial period. Int $J$ Oral Maxillofac Implants 2:77-84. 